\title{
Desenvolvimento de um Novo Sistema para Gerenciar Requisições e Laudos Periciais em Mato Grosso
}

\author{
Larissa do Carmo Silva ${ }^{1}$, Priscila de Souza Lopes ${ }^{1}$, Thiago Meirelles Ventura ${ }^{1}$, \\ Raphael de Souza Rosa Gomes ${ }^{1}$, Thaires Alves de Jesus Gonçalves ${ }^{1}$ \\ ${ }^{1}$ Instituto de Computação - Universidade Federal do Mato Grosso (UFMT) \\ Cuiabá - MT - Brasil \\ \{larissa.do.c.silva, thaires.agoncalves\}@gmail.com, \\ priscilalopes92@hotmail.com, \{thiago, rafael\}@ic.ufmt.br
}

\begin{abstract}
Criminal inspections in Mato Grosso are carried out by Perícia Oficial e Identificação Técnica de Mato Grosso (POLITEC-MT). This agency has four different applications to manage requests and expert reports, bringing difficulties in use, upgrade, data integration and performance. This work reports the analysis of this scenario and the development of a new application, which aims to facilitate POLITEC-MT's internal processes through software with good usability and efficiency.
\end{abstract}

Resumo. As perícias criminais em Mato Grosso são realizadas pela Perícia Oficial e Identificação Técnica de Mato Grosso (POLITEC-MT). Este órgão possuía quatro diferentes sistemas para gerenciar as requisições e laudos periciais, trazendo dificuldades de uso, expansão, integração de dados e desempenho. Este trabalho relata a análise desse cenário e o desenvolvimento de um novo sistema, que tem como objetivo facilitar os processos internos da POLITEC-MT por meio de um software com boa usabilidade e eficiência.

\section{Introdução}

A perícia criminal é ato característico do estado, de cunho técnico-científico, previsto no Código de Processo Penal (CPP) (BRASIL, 1941) e tem como objetivo analisar vestígios como forma de esclarecer crimes. Cabe ao perito realizar a perícia, que é o exame de algo ou alguém realizado por técnicos ou especialistas em determinados assuntos, podendo fazer afirmações ou extrair conclusões pertinentes ao processo penal (NUCCI, 2016).

Uma das formas utilizadas para fazer o pedido de perícia é por meio de uma requisição oficial. No CPP é definido em seu artigo $6^{\circ}$ que a autoridade policial deverá isolar o local para que as provas não sejam alteradas até que os peritos cheguem ao local e realizem a perícia. A perícia tem papel fundamental, permitindo uma abordagem eficiente e produzindo provas conclusivas para o caso. Assim, é necessário dar as ferramentas adequadas para que o trabalho se torne eficiente e otimizado, provendo e dando manutenção nas tecnologias necessárias para esse "fazer" científico (MISSE, 2006).

Em Mato Grosso foi institucionalizado pela Lei complementar $n^{\circ} 391$ de 27 de abril de 2010 a Perícia Oficial e Identificação Técnica de Mato Grosso (POLITEC-MT), que tem como missão realizar a perícia oficial de natureza criminal e identificação 
técnica por meio do conhecimento científico, contribuindo para a cidadania, justiça e os direitos humanos. Para que o trabalho dos servidores da POLITEC-MT possa ser eficaz são necessárias ferramentas de trabalho que atendam às demandas impostas fazendo-se necessário adequar os sistemas utilizados.

Segundo a Política Nacional de Inteligência de Segurança Pública (BRASIL, 2021), é imprescindível e urgente aperfeiçoar as tecnologias existentes. Um conhecimento completo, abrangente, preciso e oportuno, cujos dados possam ser extraídos de todas as fontes possíveis, com análise do máximo de variáveis implicadas, é o objetivo a ser atingido. Em se tratando da POLITEC-MT, há quatro sistemas relacionados ao gerenciamento de requisições e emissões de laudos periciais, e que precisam ser integrados e atualizados. Este trabalho descreve o processo de análise e proposta de solução de sistema para otimizar os processos periciais da POLITEC-MT.

\section{Análise dos Sistemas Legados}

Atualmente, a POLITEC-MT conta com quatro sistemas (MATO GROSSO, 2020) que são utilizados por usuários internos e externos, sendo eles:

- Laudos: destinado aos usuários internos e usuários assinantes da Diretoria Metropolitana de Medicina Legal e as unidades de Medicina Legal vinculadas à Diretoria de interiorização;

- Laudos V2: destinado aos usuários internos e usuários assinantes da Coordenadoria de Perícias Externas da Diretoria de Interiorização;

- Laudo Web: destinado aos usuários internos e usuários assinantes da Diretoria Metropolitana de Laboratório Forense e Coordenadoria de Perícias Internas da Diretoria Metropolitana de Criminalística;

- Politec Online: destinado a usuários internos e externos para visualização dos laudos finalizados.

Esses sistemas foram desenvolvidos à medida que as necessidades do órgão apareciam. E pelo tempo de lançamento dos sistemas, é comum haver problemas de organização e qualidade. Segundo Pressman (2011), sistemas legados podem ter como característica uma baixa qualidade, ocorrendo problemas como falta de documentação, projetos não expansíveis, códigos intrincados, entre outros. Esses problemas podem ser observados nos sistemas citados, tendo em vista que não supriam de fato a necessidade dos usuários, causando inúmeros transtornos para a realização das principais atividades.

Considerando os sistemas legados, pode ser apontado alguns dos principais problemas que motivaram a atualização: software desatualizado, lentidão para a realização de tarefas simples, baixa responsividade, sistemas não integrados entre si, e recursos se tornando limitados com o aumento da demanda.

Pressman (2011) destaca os requisitos de qualidade: funcionalidade, usabilidade, confiabilidade, desempenho e facilidade de suporte. E Nielsen (1994) relata que é necessário que um sistema tenha seu designer visível de forma que o usuário tenha facilidade em seguir o fluxo do processo de trabalho, bem como ser flexível interagindo com o usuário e trazendo mais eficiência ao trabalho que está sendo realizado por ele. Acrescenta ainda a importância das interfaces serem minimalistas, contendo as informações relevantes e indicando de forma clara o caminho a ser percorrido para 
evitar falhas. Destaca que o sistema necessita de documentação, mas não deve estar preso a isso, sendo preciso que o sistema esteja desenvolvido de uma maneira que o usuário possa utilizá-lo sem recorrer a todo tempo à explicações de terceiros ou à documentação.

Levando em consideração tais conceitos, foi possível observar que as principais deficiências dos sistemas legados se encontram principalmente nos atributos de usabilidade e desempenho. Sobre usabilidade, foi observado que em três dos quatro sistemas (Laudos V2, Laudos Web e Politec Online) os usuários eram prejudicados pela falta de atratividade e responsividade das interfaces, além da dificuldade de visualização dos campos e textos existentes. Já tendo como base o desempenho, os problemas foram verificados nos quatro sistemas, tendo em vista que o tempo de resposta a solicitações simples como autenticação, pesquisa de dados e geração de relatórios geram insatisfações aos usuários devido a demora para o atendimento dessas demandas.

Segundo Pheasant (1997) o objetivo em projetar um sistema é obter a melhor integração entre usuário e produto em relação a tarefa a ser desempenhada, é uma ciência, denominada ergonomia que objetiva adaptar o trabalho ao trabalhador e o produto ao usuário. Moraes (2010) expõe que com a evolução dos modelos de sistemas homem-máquina, é introduzido novos paradigmas, destacando questões cognitivas e de conformidade na comunicação, propondo modelagens que propiciem a qualidade e impedem ruídos na comunicação. Desta forma, surgiu a necessidade do desenvolvimento de um novo sistema, com o intuito de garantir ao usuário uma maior qualidade na realização de suas atividades. A solução desenvolvida é relatada a seguir.

\section{Solução Desenvolvida}

Dados os problemas citados anteriormente, foram tomadas medidas para que um novo sistema atenda as necessidades dos usuários e garanta sua longevidade e adaptação para evolução de acordo com realidades futuras. Exemplos de telas de um dos sistemas antigos e do novo sistema são mostrados nas Figuras 1 e 2.

Essas medidas se encontram principalmente no fato de todas as funcionalidades dos quatro sistemas (Laudos, Laudos V2, Laudos Web e Politec Online) poderem estar integradas em um único sistema, o que auxilia na manutenção, segurança e facilidade de administração do mesmo. Além disso, novas funcionalidades na manipulação das requisições para facilitar o fluxo dos processos podem ser implementadas proporcionando uma maior otimização de tempo e gestão aos usuários.

O novo sistema foi desenvolvido usando Java como linguagem de programação no backend, com o auxílio do Spring Boot. ReactJs foi utilizado para o frontend, além do sistema gerenciador de banco de dados Oracle.

Com relação às deficiências nos atributos de qualidade antes mencionados, como usabilidade, foi resolvido por meio da implementação de recursos estéticos que buscam a atenção do usuário, além de aumentar a acessibilidade descrevendo e mostrando os textos e campos de maneira clara, além de seguir os padrões de cores semânticas (para comunicar sucesso, alerta, notificações, destaques e erros), o que facilita a intuitividade do fluxo do sistema. 


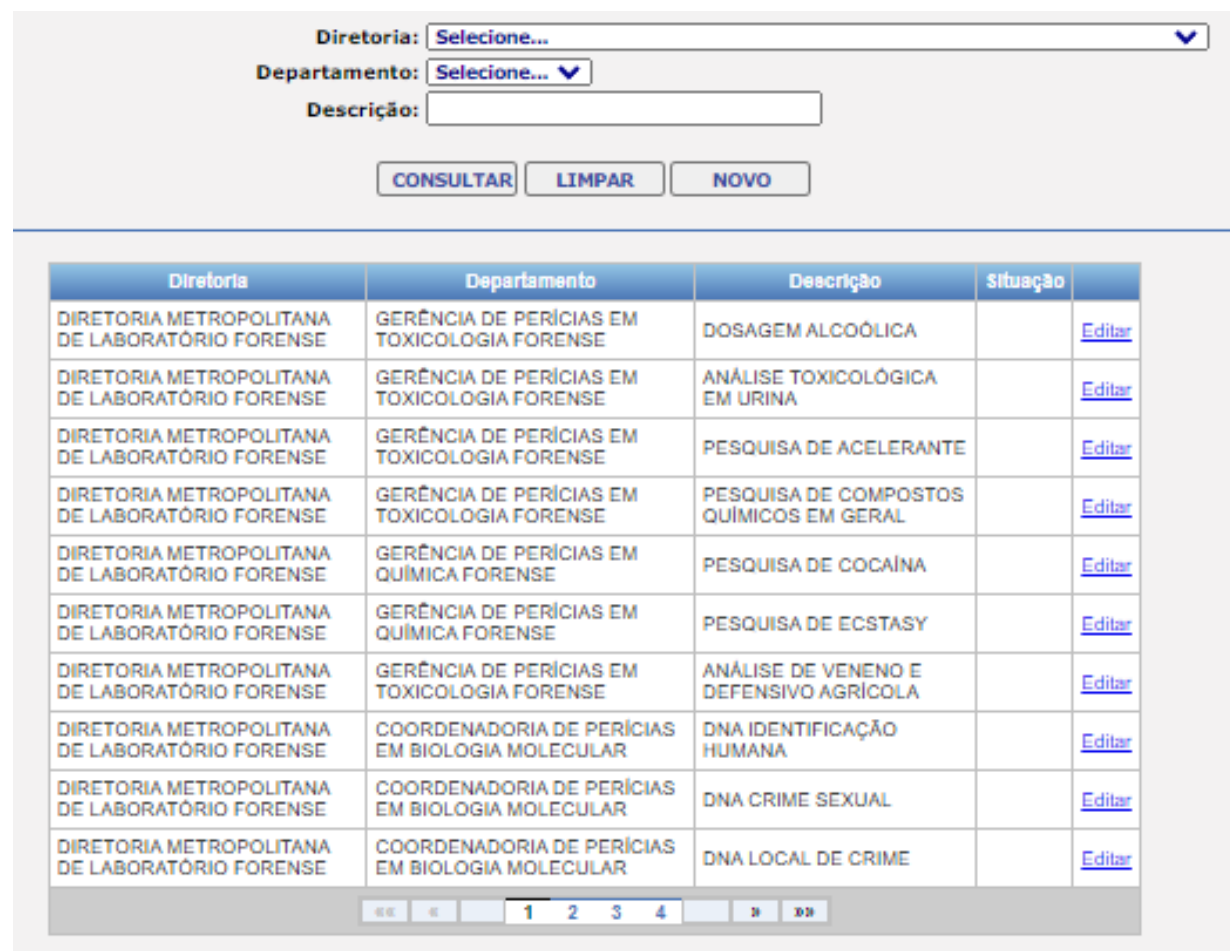

Figura 1. Tela de listagem de Natureza do Crime no sistema Laudos Web.

Natureza do Crime

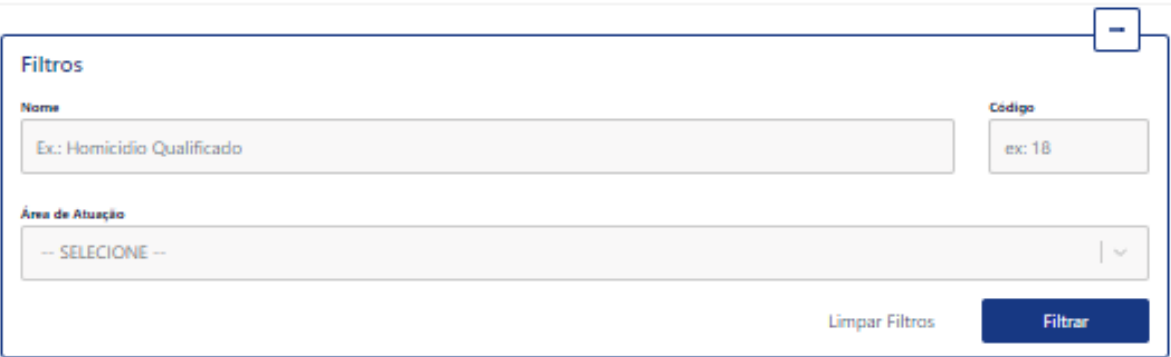

+ Adcionar

\begin{tabular}{|c|c|c|c|c|c|}
\hline Nome $=$ & Código: & Natureza Superior * & Áres de Atuaç̧aso = & & \\
\hline AUDIO E VIOEO & 01 & & CRIMINALISTICA & Editar & Remover \\
\hline СOMPUTAÇÃO FO... & 17 & & CRIMINALISTICA & Editar & Remover \\
\hline CRIMES CONTRA O... & 22 & & CRIMINALISTICA & Editar & Remover \\
\hline QUIMICA FORENSE & 60 & & LABORATORIO FOR. & Editar & Rernover \\
\hline Sribindo 4 resultados de & & & & . & 1 \\
\hline
\end{tabular}

Figura 2. Tela de listagem de Natureza do Crime no novo sistema. 
O layout do novo sistema foi feito utilizando cores relacionadas ao padrão cromático já existente na POLITEC-MT, o que pode fazer com que o usuário sinta uma maior familiaridade com o ambiente. Outra adição foi a inserção de ícones expressando mais claramente do que se trata a operação retratada, além da disposição dos botões que ocorre de forma prioritária da direita para esquerda com a cor primária indicando a ação majoritária e a secundária a ação remanescente. Ademais, foi acrescentado o atributo de responsividade, que se adequa ao dispositivo utilizado pelo usuário, dando a opção também da utilização de dispositivos móveis.

A estruturação tipográfica do sistema foi implementada de forma padronizada, utilizando fontes que fornecem uma boa visualização sendo organizadas por tamanho e cor, diferenciando títulos, subtítulos, mensagens (erro e sucesso) e textos. Após um estudo feito especificamente com um grupo de usuários selecionados de diversos setores do órgão, a elaboração das tabelas e dos filtros existentes foi realizada de acordo com as funcionalidades se adequando às necessidades dos mesmos, o que facilita na compreensão e nas consultas dos dados. Além disso, as mensagens no sistema estão dispostas de forma clara e sucinta, deixando explícito ao usuário os erros encontrados ou o sucesso nas operações.

Levando em consideração os problemas de desempenho, no novo sistema a estruturação do banco de dados ocorreu de maneira a facilitar a quantidade de consultas e retornos entre as tabelas existentes. Dessa forma, a modelagem facilitou com que as ações de busca fossem feitas de maneira otimizada, visto que os campos de pesquisa são mais específicos, o que consequentemente torna a consulta específica, melhorando com isso o tempo de resposta considerando que a busca ao banco de dados é feita com base em informações pontuais. Por fim, devido o banco de dados possuir maior rigidez nas validações das informações, foi percebido uma diminuição da necessidade de efetuar o tratamento dos dados antes de exibi-los ao usuário.

As melhorias citadas anteriormente tem por objetivo proporcionar ao usuário final uma ferramenta que irá auxiliar em seu exercício profissional, suprindo suas necessidades e atuando como um facilitador tendo em vista a grande quantidade de trâmites internos existentes na POLITEC-MT. A junção dos quatro sistemas em um único traz benefícios, como a facilidade de manutenção e expansão de funcionalidades, integração de dados para emissão de relatórios, e padronização dos processos em todos os setores do órgão.

\section{Considerações Finais}

O presente trabalho apresentou a análise e desenvolvimento de sistema que possuem o objetivo de gerenciar requisições e laudos periciais. Foram abordados os principais motivos da construção de um novo sistema, com o foco principal em facilitar a utilização dos usuários e integração de dados para geração de informações mais precisas.

Como principais pontos de mudanças, foi destacado deficiências nos atributos de qualidade, mais especificamente os de usabilidade e desempenho, os quais eram pontuados como mais recorrentes, necessitando de maior atenção. Como solução, foi feita a reformulação das interfaces, proporcionando aos usuários maior interatividade e 
acessibilidade. Além disso, as funcionalidades dos quatro antigos sistemas foram integradas em um único novo sistema, possibilitando uma manutenção mais fácil e emissão de relatórios mais precisos. Por fim, houve a adição de novas funcionalidades, incrementando o valor do sistema.

Como trabalhos futuros, há o interesse de expansão do sistema, aumentando o número de funcionalidades, integrando com outros sistemas do próprio órgão e de órgãos relacionados, e gerando dashboards dos dados coletados no sistema. É pretendido também uma análise mais aprofundada da experiência do usuário comparando os sistemas legados com o novo sistema.

\section{Agradecimentos}

Os autores deste trabalho agradecem o apoio financeiro da Fundação de Amparo à Pesquisa do Estado de Mato Grosso (FAPEMAT), processo 219559/2020.

\section{Referências}

Brasil. Decreto-Lei $\mathrm{n}^{\mathrm{o}} 3.689$, de 03 de outubro de 1941. Institui o Código de Processo Penal. Diário Oficial da União, Rio de Janeiro, 03 out. 1941.

Brasil. Decreto 10.777, de 24 de agosto de 2021. Institui a Política Nacional de Inteligência de Segurança Pública. Diário Oficial da União. Brasília, 24 de ago. 2021.

Mato Grosso. Conselho de Política Científica e Tecnológica. Portaria n 005/2020, de 01 de junho de 2020. Ratifica a implantação do sistema laudos como ferramenta oficial de registro de protocolo (requisições) e vinculação dos correspondentes laudos periciais e dá outras providências. Mato Grosso, MT, 01 jun. 2020.

Mato Grosso. Lei complementar $\mathrm{n}^{\mathbf{o}}$ 391, de 27 de abril de 2010. Dispõe sobre a institucionalização, a organização, a competência e a estrutura da Perícia Oficial e Identificação Técnica do Estado de Mato Grosso - POLITEC. Diário Oficial. Mato Grosso, 27 abr. 2010.

Misse, M. Avaliação da formação e da capacitação profissional dos peritos criminais no Brasil. Relatório final. UFRJ - NECVU, 2006.

Moraes, A., Mont'alvão, C. Ergonomia: Conceitos e aplicações. 1.ed. Rio de Janeiro : $2 \mathrm{AB}, 2010$.

Nielsen, J. Heuristic evaluation. In Nielsen, J., and Mack, R.L. (Eds.), Usability Inspection Methods, John Wiley \& Sons, New York, NY, 1994.

Nucci, G. S. Código de Processo Penal comentado. 15. ed. rev., atual. e ampl. Rio de Janeiro: Forense, 2016.

Pheasant, S. Bodyspace: Anthropometry, ergonomics and the designer of work. CRC Press, Florida, EUA, 1997. 
Pressman, R. S. Engenharia de Software: uma abordagem profissional. Tradução de Ariovaldo Griesi, Mario Moro Fecchio. 7. ed. Porto Alegre : AMGH, 2011.

Sergipe. Secretaria de Segurança Pública. Manual de Requisições da Perícia Oficial. 1. ed. Aracaju. 2018. 